\title{
Магнитные поля активных областей NOAA 11476 и NOAA 11515 во время вспышек
}

\author{
O.C. Гопасюк \\ ФГБУН “Крымская астрофизическая обсерватория РАН”, Научный, Крым, 298409 \\ olg@craocrimea.ru
}

Поступила в редакцию 15 ноября 2017 г.

\begin{abstract}
Аннотация. По данным инструмента HMI/SDO проведены исследования магнитного поля активных областей NOAA 11476 и NOAA 11515. Во время максимальной фазы вспышек M5.7/2B (NOAA 11476) и M5.3/2B (NOAA 11515) в магнитном поле активных областей появились транзиентные структуры. Эти транзиенты располагались в тени пятен, существовали в течение нескольких минут и показали пространственное и временное соответствие ядрам вспышек, наблюдаемым в $\mathrm{H}_{\alpha}$. Мы исследуем эти особенности магнитного поля и их связь с различными аспектами вспышек.

MAGNETIC FIELDS OF ACTIVE REGIONS NOAA 11476 AND NOAA 11515 DURING FLARES, by O.S. Gopasyuk. We investigate the magnetic fields of active regions NOAA 11476 and NOAA 11515 using the HMI/SDO data. The transient features appeared in the magnetic fields of active regions during the peak phase of the M5.7/2B (NOAA 11476) and M5.3/2B (NOAA 11515) flares. These transients were located in the sunspot umbrae and existed for a few minutes. The transient features showed spatial and temporal correspondence with the $\mathrm{H}_{\alpha}$ flare kernels. We examine these features and their relationship with various aspects of the flares.
\end{abstract}

Ключевые слова: Солнце, фотосфера, магнитные поля, вспышки

\section{1 Введение}

Во время мощных вспышек Х- и М-класса происходят значительные изменения фотосферного магнитного поля (Ванг, 1992; Ванг и др., 1994; Косовичев, Жаркова, 2001; Судол, Харви, 2005; Маура и др., 2012; Бурцева и др., 2015). Наблюдения показывают два вида изменений магнитного поля во время вспышек. Первый вид - это необратимые изменения измеренного магнитного поля от состояния перед вспышкой до состояния после вспышки. Обычно наблюдаются в виде появления или исчезновения потока и рассматриваются как реальные изменения магнитного поля. Второй вид “транзиенты” - наблюдаются только во время вспышек, при этом измеренные магнитные поля возобновляют свое предвспышечное состояние после события. В некоторых случаях магнитная аномалия принимает форму обращения знака, т. е. измеренные магнитные поля временно меняют свои полярности на противоположные.

В данной работе мы исследуем транзиентные структуры магнитного поля двух активных областей и связь этих структур с различными аспектами вспышек. 


\section{2 Данные наблюдения и их анализ}

Были использованы данные космических инструментов HMI (Шоу и др., 2012), установленного на борту SDO, и инструмента GOES, а также данные наземных солнечных обсерваторий Концельхое (KCO), Удайпур (УСО) и Мауна Лоа (МСО).

$\mathrm{HMI}$ SDO получает магнитограммы и изображения в континууме полного диска Солнца в фотосферной линии поглощения Fe I $6173.3 \AA$ с пространственным и временным разрешением $0.5^{\prime \prime}$ пиксель ${ }^{-1}$ и 45 с соответственно. Магнитограммы и изображения в континууме активных областей были получены на HMI/SDO 10 мая 2012 г. во время вспышки M5.7/2B (NOAA 11476) и 4 июля 2012 г. во время вспышки M5.3/2B (NOAA 11515).

Для определения местоположения вспышек были использованы наземные хромосферные $\mathrm{H}_{\alpha}$ наблюдения, полученные на КСО, а также данные обсерваторий УСО и МСО, входящих в сеть GONG. Пространственное разрешение этих данных $1^{\prime \prime}$ пиксель ${ }^{-1}$.

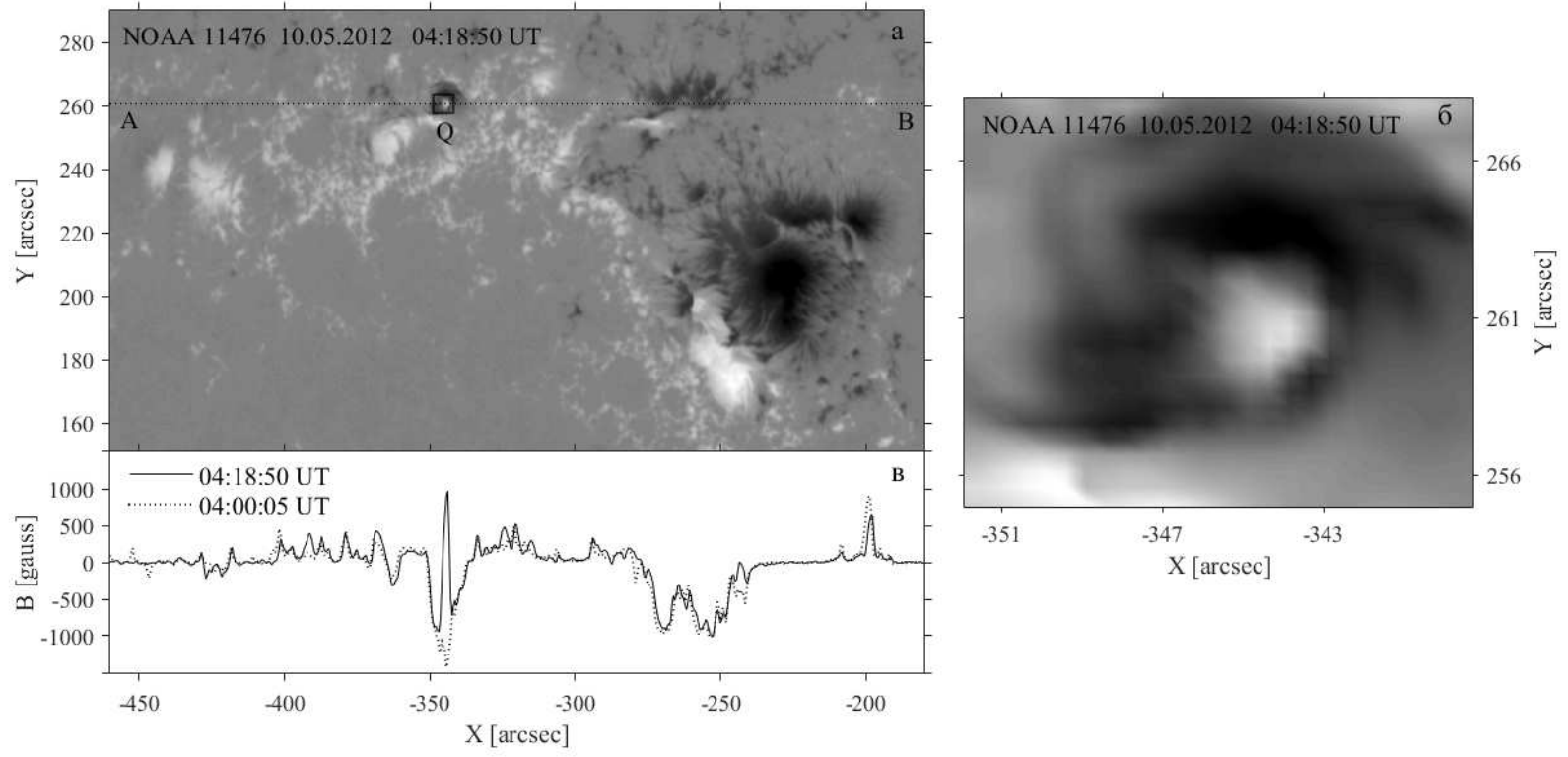

Рис. 1. (а) - Магнитограмма активной области NOAA 11476, полученная на HMI/SDO 10 мая 2012 г. во время максимальной фазы вспышки M5.7/2B. Прямоугольником “Q” выделена область, в которой во время максимальной фазы вспышки наблюдалась инверсия знака магнитного поля. (б) - в увеличенном масштабе магнитограмма области “Q”. (в) - магнитный поток вдоль линии АВ перед вспышкой (пунктирная кривая) и во время максимальной фазы вспышки (сплошная кривая). Белые/черные области в магнитограммах представляют положительные/отрицательные полярности

За время прохождения по диску Солнца активные области (AO) NOAA 11476 и NOAA 11515 показали высокую вспышечную активность. 10 мая 2012 г. в NOAA 11476 произошло 17 вспышек С- и М-класса. Активная область находилась в южном полушарии и на момент вспышки M5.7/2B была вблизи центрального меридиана на долготе Е22. В NOAA 115154 июля 2012 г. произошло 15 вспышек С- и M-класса. АО находилась в северном полушарии, во время вспышки M5.3/2B она была в западном полушарии на долготе W18.

На рис. 1а и рис. 2а представлены магнитограммы активных областей NOAA 11476 и NOAA 11515 во время максимальной фазы вспышек M5.7/2B и M5.3/2B соответственно. Прямоугольником выделены области “Q”, в которых в момент максимальной фазы вспышек наблюдалась 
аномалия магнитного поля. На рис. $1 б$ и рис. $2 б$ область магнитной аномалии “Q” представлена в более крупном масштабе. Магнитные аномалии в обеих активных областях появились в тени пятен. В сильных магнитных полях отрицательной полярности возникли области положительной полярности. B NOAA 11476 аномалия наблюдалась в тени пятна простой конфигурации, в то время как в NOAA 11515 пятно было дельта-конфигурации. Для обеих активных областей был исследован маг-

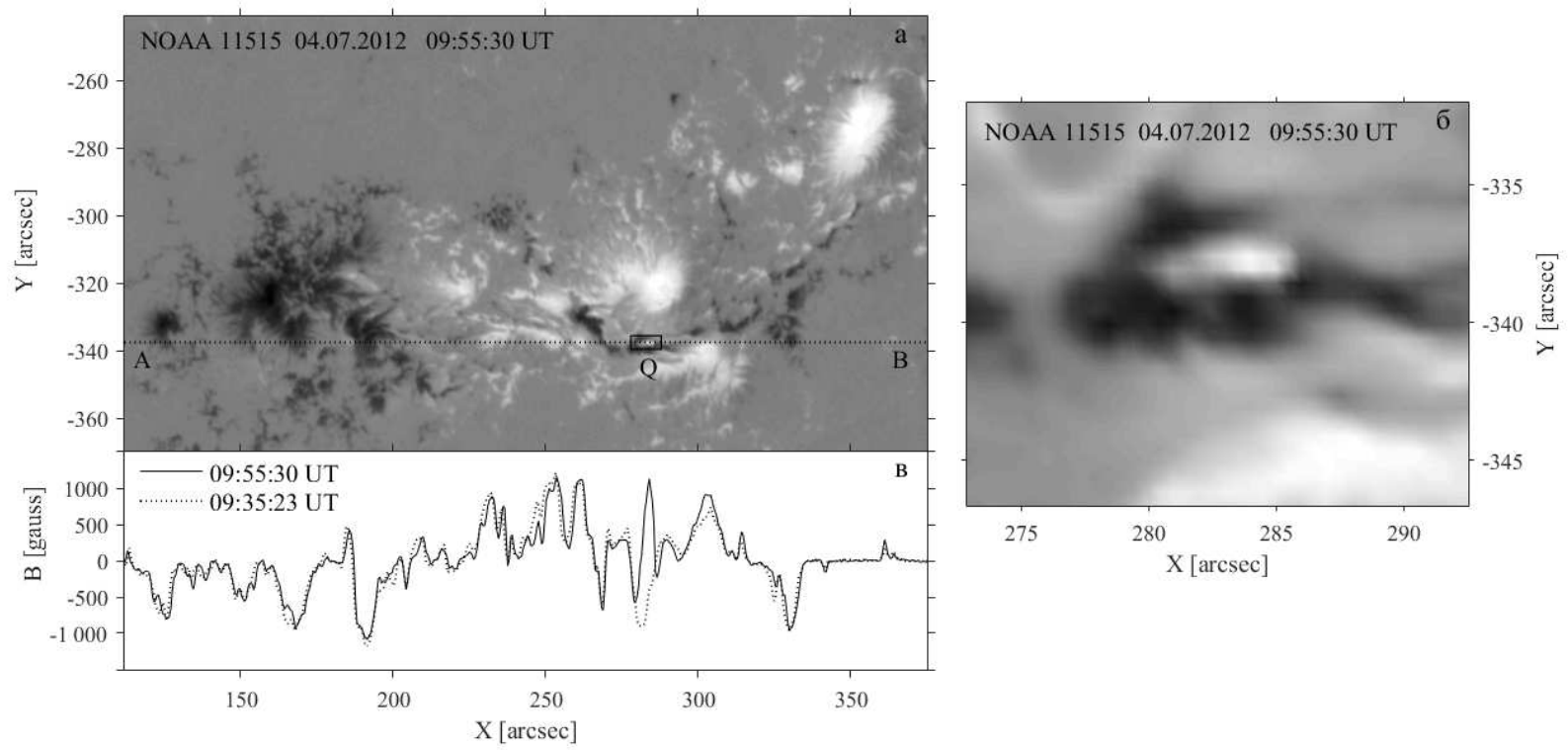

Рис. 2. (а) - Магнитограмма активной области NOAA 11515, полученная на HMI/SDO 4 июля 2012 г. во время максимальной фазы вспышки M5.3/2B. Прямоугольником “Q” выделена область, в которой во время максимальной фазы вспышки наблюдалась инверсия знака магнитного поля. (б) - в увеличенном масштабе магнитограмма области “Q”. (в) - магнитный поток вдоль линии AB перед вспышкой (пунктирная кривая) и во время максимальной фазы вспышки (сплошная кривая). Белые/черные области в магнитограммах представляют положительные/отрицательные полярности

нитный поток вдоль горизонтальной линии $\mathrm{AB}$, проведенной через область “Q”, до вспышки и во время максимальной фазы вспышки. Профили магнитного потока вдоль линии АВ в области “Q” для NOAA 11476 и NOAA 11515 показаны на рис. 1в и рис. 2в, где пунктирная и сплошная кривые представляют магнитный поток до вспышки и во время ее максимальной фазы соответственно. Профиль магнитного потока в предвспышечной и максимальной фазах совпали во всех точках вдоль АВ, за исключением области аномалии “Q”. В обоих случаях в области аномалии напряженность магнитного поля менялась примерно от -1000 Гс (в момент до вспышки) до +1000 Гс (во время максимальной фазы вспышки).

Для исследования вариаций со временем магнитного потока аномалии были построены пространственно-временные карты магнитного поля вдоль АВ. Такие же пространственно-временные карты были построены для изображений $\mathrm{AO}$ в линии $\mathrm{H}_{\alpha}$. На рис. 3 представлены пространственновременные карты магнитного поля области “Q” вдоль линии $\mathrm{AB}$ с соответствующими $\mathrm{H}_{\alpha}$ вспышечными интенсивностями. Эти карты показывают четкую структуру магнитных транзиентов. В обеих активных областях магнитная аномалия располагалась в узлах вспышек и не меняла местоположение в течение всего времени своего существования. Отметим, что площадь, занимаемая магнитной аномалией, существенно меньше площади узла вспышки в $\mathrm{H}_{\alpha}$. 

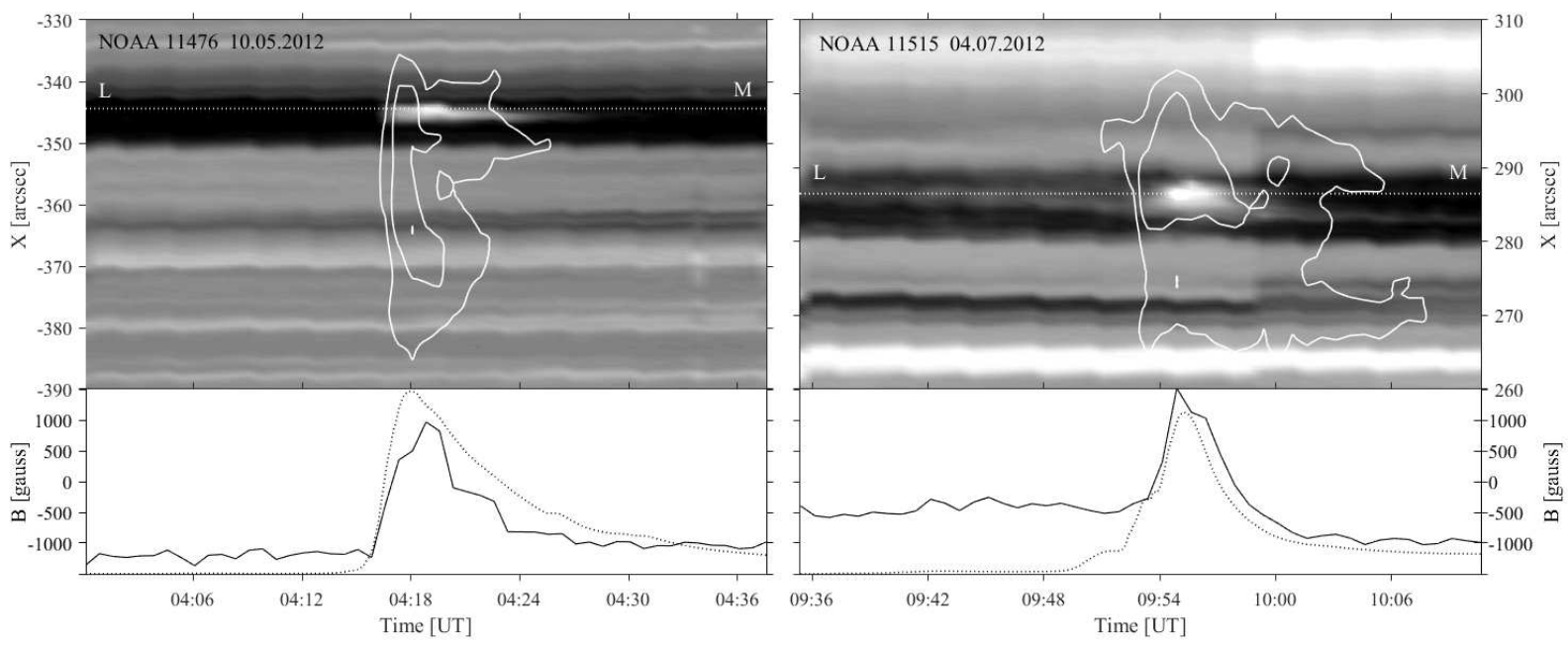

Рис. 3. Пространственно-временные карты магнитного потока в области "Q" NOAA 11476 (верхняя панель, слева) и NOAA 11515 (верхняя панель, справа) вдоль линии АВ (рис. 1а и рис. 2а) с соответствующими $\mathrm{H}_{\alpha}$ вспышечными интенсивностями (белые сплошные кривые). На нижних панелях показаны профили магнитного потока вдоль линии LM (сплошная кривая) и интегрального потока излучения в мягком рентгене по данным GOES-15 в диапазоне 1.0-8.0 А̊ (пунктирная кривая)

На нижней панели рис. 3 показаны временные изменения магнитного потока вдоль линии LM и потока мягкого рентгеновского излучения в диапазоне 1-8 ̊, полученного на GOES-15. В обоих случаях рентгеновское излучение начинает расти на 1-2 минуты раньше магнитного потока. Максимум напряженности поля в магнитной аномалии NOAA 11476 наступает на 2 минуты позже максимума рентгеновского излучения. В NOAA 11515 напряженность в магнитной аномалии достигает своего максимального значения практически одновременно с максимумом рентгеновского излучения.
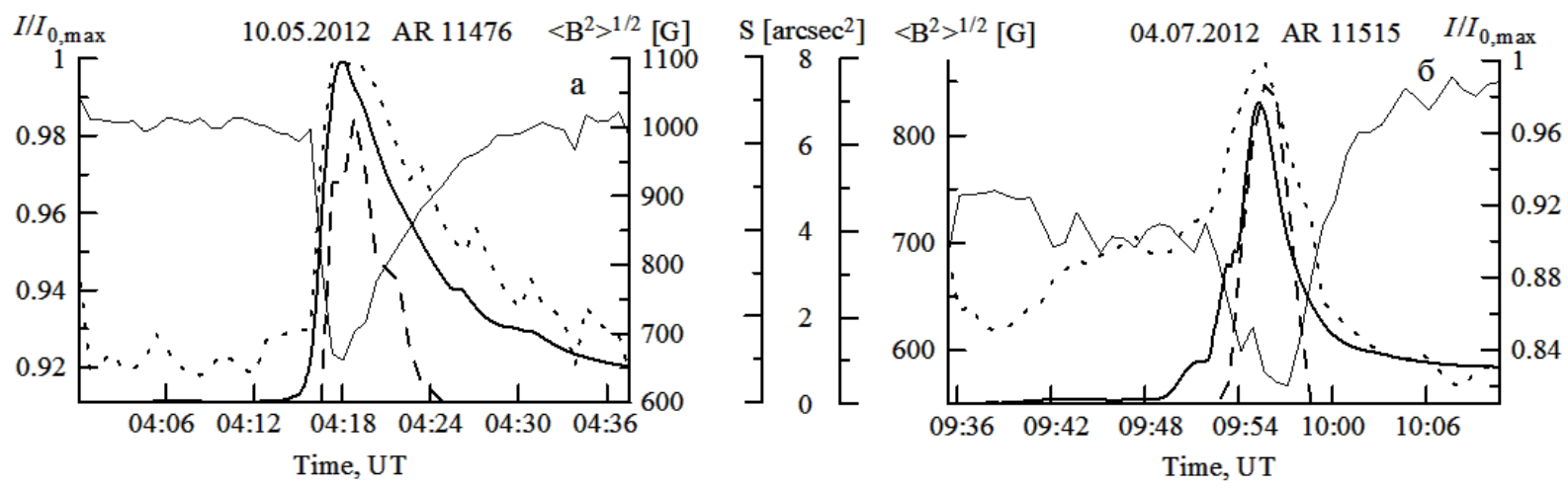

Рис. 4. Временные вариации в области “Q” активных областей NOAA 11476 (а) и NOAA 11515 (b) rms магнитного поля, $<B^{2}>^{1 / 2}$ (тонкая сплошная кривая), средней относительной интенсивности в континууме, $I / I_{0, \max }$ (пунктирная кривая), площади магнитной аномалии $S$ (штриховая кривая) и интегрального потока мягкого рентгеновского излучения по GOES-15 (жирная сплошная кривая) 
Для обеих $\mathrm{AO}$ были определены некоторые характеристики магнитной аномалии. На рис. 4 показаны изменения со временем средней квадратичной плотности магнитного потока $<B^{2}>^{1 / 2}$, площади магнитной аномалии $S$, средней относительной интенсивности в линии Fe I $6173.3 \AA I / I_{0, \max }$ и потока мягкого рентгеновского излучения в диапазоне 1-8. . Все кривые хорошо коррелируют между собой. Изменение магнитного поля в обеих АО сопровождалось сильным импульсным увеличением средней относительной интенсивности. Так, для NOAA 11476 увеличение составило $8 \%$, а для NOAA $11515-17 \%$.

В NOAA 11476 магнитная аномалия существовала 8 минут. Максимальная площадь аномалии составила 7 квадратных секунд дуги. В NOAA 11515 транзиентная структура наблюдалась в течение 6 минут. Ее максимальная площадь составила также 7 квадратных секунд дуги.

\section{3 Выводы}

По данным инструмента HMI/SDO проведены исследования магнитного поля активных областей NOAA 11476 и NOAA 11515. Во время максимальной фазы вспышек M5.7/2B (NOAA 11476, 10 мая 2012 г.) и M5.3/2B (NOAA 11515, 4 июля 2012 г.) в магнитном поле обеих активных областей появились транзиентные структуры. Этот феномен до сих пор не совсем понятен, остаются вопросы относительно физического механизма возникновения таких структур. Они могут иметь чисто инструментальную природу и быть вызваны изменением профиля спектральной линии вследствие бомбардировки фотосферной плазмы ускоренными электронами (Дин и др., 2002). В то же время это предположение не подтверждается расчетами Косовичева и Жарковой (2001).

Нами были получены следующие характеристики наблюдаемых магнитных аномалий. В сильных магнитных полях отрицательной полярности возникли области сильных полей положительной полярности. Напряженности магнитного поля менялись от -1000 Гс в предвспышечный момент до +1000 Гс во время максимальной фазы вспышек. Между изменениями потока мягкого рентгена в диапазоне 1-8 , магнитного потока в аномалии, ее площади и изменениями средней относительной интенсивности в фотосферной линии наблюдается хорошая корреляция. Изменение магнитного поля в транзиентных структурах сопровождалось сильным импульсным увеличением средней относительной интенсивности (до $17 \%$ ). Эти структуры располагались в тени пятен, существовали в течение 6-8 минут и показали пространственное и временное соответствие ядрам вспышек, наблюдаемым в $\mathrm{H}_{\alpha}$. В обоих случаях площадь, занимаемая аномалиями, составила примерно 7 квадратных секунд дуги, что существенно меньше площади узла $\mathrm{H}_{\alpha}$-вспышки.

Аномальное изменение знака магнитной полярности во время мощных вспышек наблюдалось и по данным инструментов GONG и MDI, которые в своих измерениях используют линию Ni I (см. например, Косовичев, Жаркова, 2001; Маура, Амбастха, 2009), тогда как данные НМI для вспышек M5.7 и M5.3, исследованные в этой статье, основаны на измерениях в линии Fe I. Таким образом, транзиентные структуры, возникающие во время максимальной фазы вспышек, по-видимому, не связаны с линией и инструментом, используемыми в измерениях. Следовательно, наблюдаемые магнитные транзиенты можно рассматривать как наблюдательные характеристики физических процессов, происходящих в фотосфере активных областей во время максимальной фазы вспышки.

Благодарности. Эта работа использует данные GONG NSO под управлением AURA в соответствии с соглашением о сотрудничестве с NSF и при дополнительной финансовой поддержке NOAA, NASA и ВBC США. Автор благодарен научной команде NASA/SDO и HMI за возможность доступа к базам данных по сети Интернет. Данные о мягких рентгеновских потоках GOES доступны в NASA/GSFC Solar Data Analysis Center (SDAC). Работа выполнена при частичной поддержке гранта РФФИ 1602-00221 A.

\section{Литература}

Бурцева и др. (Burtseva O., Martínez-Oliveros J.C., Petrie G.J.D., Pevtsov A.A.) // Astrophys. J. 2015. V. 806. P. 173.

Ванг (Wang H.) // Solar Phys. 1992. V. 140. P. 85.

Ванг и др. (Wang H., Ewell Jr.M.W., Zirin H., Ai G.) // Astrophys. J. 1994. V. 424. P. 436. 
Дин и др. (Ding M.D., Qiu J., Wang H.) // Astrophys. J. 2002 V. 576. L. 83.

Косовичев, Жаркова (Kosovichev A.G., Zharkova V.V.) // Astrophys. J. 2001. V. 550. P. L105.

Маура, Амбастха (Maurya R.A., Ambastha A.) // Solar Phys. 2009. V. 258. P. 31.

Маура и др. (Maurya R.A., Vemareddy P., Ambastha A.) // Astrophys. J. 2012. V. 747. P. 134.

Судол, Харви (Sudol J.J., Harvey J.W.) // Astrophys. J. 2005. V. 635. P. 647.

Шоу и др. (Schou J., Scherrer P.H., Bush R.I., et al.) // Solar Phys. 2012. V. 275. P. 229. 